DOI: https://doi.org/10.11144/Javeriana.upsy17-3.tops

\title{
Tríada oscura de personalidad, satisfacción laboral y cinismo organizacional: un modelo estructural ${ }^{*}$
}

\section{Dark Triad of Personality, Job Satisfaction and Organizational Cynicism: A Structural Model}

\author{
Solana SAlessi ${ }^{\mathrm{a}}$ \\ Consejo Nacional de Investigaciones Científicas y \\ Técnicas, Argentina \\ ORCID: http://orcid.org/0000-0001-9496-9493 \\ Alicia Omar \\ Consejo Nacional de Investigaciones Científicas y \\ Técnicas, Argentina
}

a Autor de correspondencia. Correo electrónico: solanasalessi@gmail.com

Para citar este artículo: Salessi, S., \& Omar, A. (2018). Tríada oscura de personalidad, satisfacción laboral y cinismo organizacional: un modelo estructural. Universitas Psychologica, 17(3), 1-12. https://doi.org/1 0.11144/Javeriana.upsy17-3.tops

\section{RESUMEN}

Se analizó el impacto del cinismo organizacional sobre las relaciones entre la tríada oscura (maquiavelismo, narcisismo, psicopatía) y la satisfacción laboral, en una muestra de 343 trabajadores argentinos. Se plantearon hipótesis sobre relaciones directas e indirectas que fueron probadas mediante análisis de ecuaciones estructurales. Los resultados confirmaron que la tríada predice el cinismo (maquiavelismc $\Upsilon=0.39, \mathrm{p}<0.01$; psicopatía $\Upsilon=0.22, p<0.01$; narcisismo $\Upsilon=0.14$; $p<0.05$ ), y que el cinismo mediatiza completamente las relaciones entre la tríada y la satisfacción laboral (maquiavelismo: $z=-4.51, p<0.001$; psicopatía: $z=-3.24, p<0.001$; narcisismo: $z=-1.78, \mathrm{p}<0.05)$. Se discuten las implicancias prácticas de los hallazgos y se sugieren nuevas líneas de investigación futura.

Palabras clave

tríada oscura; cinismo organizacional; satisfacción laboral; modelo explicativo.

\begin{abstract}
The impact of organizational cynicism on the relationship between dark personality traits (Machiavellianism, narcissism, and psychopathy) and job satisfaction was analysed. It was study a sample of 343 Argentinean workers. Hypotheses on direct and indirect relationships were tested by structural equation modelling. The results confirmed that the dark triad predicts cynicism (machiavellianism $\Upsilon=0.39, p<0.01$; psychopathy: $\Upsilon=0.22, p<0.01$; narcissism: $\Upsilon=0.14 ; p<0.05)$; and that cynicism fully mediates the relationship between the dark side and job satisfaction (machiavellianism: $z=-4.51, p<0.001$; psychopathy: $z=-3.24, p<$ 0.001 ; narcissism: $z=-1.78, p<0.05)$. Practical implications of findings are discussed and new lines for future studies are suggested.

Keywords

dark triad; organizational cynicism; job satisfaction; explanatory model.
\end{abstract}


El nuevo milenio trajo consigo nuevos desafíos para los especialistas en comportamiento organizacional. Tras el protagonismo inicial de las capacidades y fortalezas positivas, en los últimos años el estudio de las facetas más sombrías de la personalidad se ha trasladado a un lugar central en el escenario científico. En este sentido, la contribución que las características negativas de la personalidad hacen a la emergencia y desarrollo de algunas conductas organizacionalmente perjudiciales comienza a ser objeto de especial atención (Guenole, 2014; Linstead, Maréchal, \& Griffin, 2014; Palaiou, Zarola, \& Furnham, 2016). Bajo el epíteto "personalidad oscura" se hace referencia a un conjunto ecléctico de características comprendidas entre los rasgos "normales" (luminosos) y los "anómalos". Tales rasgos se ubican por debajo del umbral clínico y no suponen una alteración psicopatológica (Spain, Harms, \& Lebreton, 2013). De hecho, las características oscuras de la personalidad no implican necesariamente obstáculos o dificultades en el funcionamiento laboral cotidiano. Sin embargo, bajo condiciones adversas pueden ocasionar resultados que perjudican, por ejemplo, la calidad de las relaciones interpersonales, el rendimiento del empleado y el logro de las metas organizacionales (Furnham, Richards, \& Paulhus, 2013).

Se han ofrecido diferentes respuestas sobre cuántos rasgos integran el lado oscuro de la personalidad. No obstante, el grueso de la investigación contemporánea se ha concentrado en una peculiar constelación de características (narcicismo, maquiavelismo y psicopatía) que, en conjunto, conforman la denominada "tríada oscura" (Paulhus, 2014). Se trata de tres variables de personalidad conceptualmente distintas, desde el momento que el maquiavelismo se caracteriza por la manipulación estratégica de los demás; la psicopatía, por la frialdad emocional y la tendencia a la provocación; en tanto que el narcicismo, por el egocentrismo, la grandiosidad y las fantasías de poder ilimitado. Sin embargo, más allá de estas particularidades, se ha demostrado que comparten elementos comunes tales como insensibilidad interpersonal, falta de empatía, ausencia de humildad, deshonestidad, manipulación y creencias en su propia superioridad (Jones \& Figueredo, 2013; Jones \& Paulhus, 2017; Stead \& Fekken, 2014).

La tendencia actual se orienta a estudiar el impacto de esta tríada oscura sobre actitudes y comportamientos en el trabajo diferentes a los clásicamente analizados (estilos de liderazgo disfuncionales, comportamientos contraproducentes, acoso psicológico, etc.). Asimismo, los especialistas (Furnham et al., 2013; Spain et al., 2013) insisten en la necesidad de explorar tanto potenciales moderadores susceptibles de atenuar el impacto adverso de estos rasgos de personalidad, así como a identificar posibles mediadores entre ellos y diferentes consecuencias organizacionales. En línea con esta agenda, el objetivo del presente estudio fue analizar si la tríada oscura de personalidad predice las actitudes cínicas de los empleados hacia sus organizaciones y si, a su vez, el cinismo actúa como un mediador entre tales rasgos de personalidad y la (in)satisfacción en el lugar de trabajo.

\section{La tríada oscura y el cinismo organizacional}

El cinismo organizacional (CO) ha sido formalmente definido (Brandes \& Das, 2006) como una actitud negativa del empleado hacia su organización. Los cínicos están convencidos de que las organizaciones en las que trabajan son inescrupulosas y carecen de integridad y responsabilidad social. Consideran que la empresa les impide participar en los procesos de toma de decisiones y que las acciones implementadas por la gerencia se basan en motivaciones ocultas que pueden perjudicarlos. Sumidos en un estado de suspicacia e incertidumbre permanentes, no logran establecer un vínculo afectivo positivo con la organización empleadora, siendo proclives a involucrarse en comportamientos negligentes hacia su trabajo y contraproducentes hacia sus organizaciones. En este sentido, se ha informado (Aly, Ghanem, 
\& El-Shanawany, 2016; Chiaburu, Peng, In-Sue, Banks, \& Lomeli, 2013; Thundiyil, Chiaburu, InSue, Banks, \& Peng, 2014) que altos niveles de $\mathrm{CO}$ se asocian negativamente con identificación laboral, conductas de ciudadanía organizacional y compromiso organizacional, así como con mayor resistencia al cambio, ausentismo e intenciones de desvinculación. A su vez, el mantenimiento de esta actitud en el tiempo hace que los empleados se transformen en víctimas de su propio recelo y decepción, con los consabidos efectos colaterales asociados a este tipo de emociones tóxicas (Salessi \& Omar, 2014a).

El análisis crítico de la literatura sobre $\mathrm{CO}$ indica que los mayores esfuerzos han estado orientados a la identificación de sus antecedentes contextuales. De allí que injusticias organizacionales, políticas de reestructuración y reducción de personal, así como estrategias ineficaces de comunicación interna y escaso apoyo organizacional (Chiaburu et al., 2013; Thundiyil et al., 2014), han sido reiteradamente señaladas como variables explicativas del CO. Si bien Andersson citada en Brandes y Das (2006) ha sugerido que el $\mathrm{CO}$ podría tener una base disposicional, los esfuerzos empíricos invertidos en comprobar la existencia de un nexo entre el cinismo y las diferencias individuales han sido exiguos. Frente a este vacío empírico, el presente estudio se orienta a explorar las potencialidades de los rasgos que conforman la tríada oscura como predictores del $\mathrm{CO}$.

En función de las características que definen al CO sería lícito presuponer que la combinación de rasgos negativos subsumida en la tríada oscura podría asociarse a una mayor frecuencia de esta actitud. Al respecto, por ejemplo, algunos autores (Furnham et al., 2013; Jones \& Paulhus, 2017) han subrayado que los maquiavélicos son esencialmente cínicos, y que, junto a los psicópatas, son quienes albergan mayores sospechas sobre las motivaciones subyacentes a las acciones de los demás. Los narcisistas, en tanto, a raíz de su autoconcepto excesivamente elevado y la convicción de ocupar un sitial preferencial, suelen desafiar el statu quo y las relaciones de poder establecidas. En virtud de estas notas distintivas, sería esperable que maquiavélicos y psicópatas desarrollen expectativas negativas sobre su entorno laboral, similares a las que se encuentran en los cimientos del CO (Chiaburu et al., 2013). Asimismo, la astucia y suspicacia que los caracteriza les permitiría percibir con mayor agudeza las contradicciones entre el discurso y las acciones de la organización, retroalimentando positivamente su desconfianza. De manera similar, podría presuponerse que el sentido de grandiosidad que define a los narcisistas los inclinaría a sentirse moralmente superiores, aspecto que ya fue señalado por Dean, Brandes y Dharwadkar citados en Brandes y Das (2006), en su caracterización del CO. Por su insaciable pretensión de ocupar posiciones de liderazgo, los narcisistas podrían sentirse fácilmente damnificados por el incumplimiento de las promesas (principalmente de aquellas que afectan directamente su estatus y prestigio dentro de la organización, tales como ascensos $\mathrm{y}$ promociones) y proclives a experimentar emociones negativas (frustración, enojo, etc.), tales como las que alimentan a las actitudes cínicas (Salessi \& Omar, 2014a). Frente a este cuadro de situación, sería factible conjeturar que: (1) el alto grado de desconfianza y malicia propio de los maquiavélicos motivaría comportamientos displicentes y negativos hacia la organización; (2) el egocentrismo típico de los narcisistas propiciaría críticas destinadas a acentuar la insuficiencia y el fracaso de la organización; (3) la hostilidad y frialdad emocional que caracteriza a los psicópatas, se asociaría a la mayor reticencia a establecer un vínculo afectivo con la organización, lo que sumado a sus tendencias a la provocación y la burla explicarían las conductas típicas de sarcasmo y humor cínico, bajo la forma de ridiculización de los valores, políticas y prácticas organizacionales. Con base en tales consideraciones, se hipotetiza que:

Hipótesis 1: Maquiavelismo, narcisismo y psicopatía emergerán como predictores de $\mathrm{CO}$. 


\section{Tríada oscura e insatisfacción laboral: el CO como mecanismo explicativo}

La satisfacción laboral, como actitud que se va consolidando por el hecho de realizar un trabajo agradable dentro del ámbito de una organización que resulta atractiva y por el que se recibe una serie de compensaciones acordes con las expectativas, es considerada como producto de la congruencia entre el puesto y las características personales del empleado (Judge, Weiss, Kammeyer-Mueller, \& Hulin, 2017). De allí que, desde la irrupción del constructo en el ámbito científico, sus raíces disposicionales han sido objeto de diversos estudios. Si bien están bien establecidas las relaciones entre los rasgos "luminosos" (usualmente conceptualizados a través de las taxonomías de la personalidad normal tales como la de los Cinco Grandes) y la satisfacción laboral ( Sundstrom, Lounsbury, Gibson, \& Huang, 2016), el impacto de los rasgos sombríos está menos documentado en la bibliografía. Por lo que se impone la necesidad de llenar este vacío empíricoconceptual desarrollando estudios en esta dirección.

Los rasgos que definen la tríada oscura a menudo se asocian con la presencia de mayor afectividad negativa, por lo que se podía esperar una vinculación negativa con experiencias placenteras tales como satisfacción laboral (Furnham et al., 2013). Al mismo tiempo, tales rasgos están frecuentemente relacionados con mayores niveles de conflicto interpersonal con los supervisores y compañeros de trabajo, por lo que sería previsible que las evaluaciones de su lugar de trabajo tiendan a ser desfavorables (Palaiou et al., 2016; Spain et al., 2013). Más allá de las especulaciones teóricas, las evidencias empíricas publicadas hasta la fecha son exiguas y los resultados acerca de la dirección de las relaciones distan de ser concluyentes, particularmente, en el caso del narcisismo. En este sentido, por ejemplo, mientras que algunos estudios (Michel \& Bowling, 2013; Spurk, Keller, \& Hirschi, 2016) han comunicado efectos principales sobre la satisfacción laboral; otros investigadores (Hirschi \& Jaensch, 2015) han informado que el narcisismo tiene efectos indirectos a través de las creencias de autoeficacia. Por lo tanto, frente a un panorama signado por la inconsistencia y la contradicción entre los hallazgos, es lícito reexaminar las relaciones entre la tríada oscura de personalidad y el grado de satisfacción de los trabajadores, así como interrogarse por el papel que el CO desempeña en este hipotético escenario. Los resultados comunicados por O'Boyle, Forsyth, Banks y McDaniel (2012) subrayan la existencia de efectos directos, aunque pequeños, de la personalidad oscura sobre el comportamiento laboral; en tanto que recientes publicaciones (Jonason, Weeb, \& Li, 2015; Palmer, Komarraju, Carter, \& Karau, 2017; Smith \& Webster, 2017) adelantan la posibilidad que el impacto de la tríada oscura ocurra a través de terceras variables. Con base en estos referentes empíricos, se conjetura que:

Hipótesis 2: el CO mediará totalmente las relaciones entre maquiavelismo, narcisismo, psicopatía y satisfacción laboral. De modo que el impacto negativo de la tríada oscura sobre la satisfacción dependerá de la presencia de actitudes cínicas hacia la organización.

\section{Método}

\section{Participantes}

La muestra estuvo conformada por 343 trabajadores argentinos (192 varones y 151 mujeres). La edad promedio de los participantes fue de 33.06 años $(D E=7.98)$ y la antigüedad laboral media de 5.77 años $(D E=7.51)$. El $46.9 \%$ de los sujetos tenía estudios superiores (terciarios o universitarios). El $34.4 \%$ pertenecía al sector de servicios (incluyendo actividades en el ámbito de la salud, la educación y la administración pública), el 29.7 \% trabajaba en el sector industrial y el $35.9 \%$ se desempeñaba en la actividad comercial. 


\section{Instrumentos}

Tríada oscura. Fue evaluada mediante la adaptación argentina (Salessi \& Omar, en prensa) de la escala homónima desarrollada por Jones y Paulhus (2014). El instrumento está integrado por 27 ítems, distribuidos en tres subescalas: maquiavelismo (p. ej.: "La mayoría de las personas pueden ser manipuladas"; $\alpha=$ 0.74), narcisismo (p. ej.: "Exijo que me traten con el respeto que merezco"; $\alpha=0.76)$ y psicopatía (p. ej.: "Podría decir cualquier cosa con tal de conseguir lo que quiero"; $\alpha=-0.78)$. Cada ítem es valorado sobre una escala tipo Likert de 5 puntos, que va desde 1 (totalmente en desacuerdo) hasta 5 (totalmente de acuerdo).

Cinismo organizacional. Se exploró mediante la adaptación argentina (Salessi \& Omar, 2014b) de la Escala de Cinismo Organizacional. El instrumento se compone de 10 ítems que exploran pensamientos, emociones y conductas típicamente cínicas (p. ej.: "Creo que mi jefe dice una cosa y hace otra"; $\alpha=0.89$ ), con formato Likert de 5 puntos, variando de 1 (nunca) a 5 (siempre).

Satisfacción laboral. Fue medida con la adaptación argentina (Salessi \& Omar, 2016) de la Escala de Satisfacción Laboral Genérica. Esta escala proporciona un puntaje global de satisfacción con el trabajo a través de siete ítems (p. ej.: "Considerándolo en términos generales tengo un buen trabajo"; $\alpha=0.86$ ), con formato Likert de 5 puntos, variando de 1 (totalmente en desacuerdo) a 5 (totalmente de acuerdo).

Deseabilidad social. Como mecanismo de validez interna se incluyó una escala para medir la tendencia a mostrar una imagen mejorada de uno mismo. La deseabilidad social fue evaluada mediante la escala L (del inglés lie: mentira) del Cuestionario EPQ de Personalidad de Eysenck adaptado para la Argentina por Omar (1988). Se utilizaron 10 ítems (p. ej.: "iTodos mis hábitos son buenos y deseables?”; $\alpha=0.79$ ) con formato de opción forzada Sí/No.

Variables control. En la ejecución de los análisis previstos, se controló el efecto del sexo, la edad y la antigüedad laboral, ya que algunas investigaciones han señalado que los varones son proclives a un mayor número de comportamientos y actitudes negativas y que los empleados de más edad y antigüedad informan mayores niveles de satisfacción laboral (Salessi \& Omar 2014b, 2016).

\section{Procedimiento}

Todos los participantes fueron contactados en sus respectivos lugares de trabajo, previa autorización de los directivos organizacionales. Con cada organización participante se pautaron días y horarios para efectuar la recolección de los datos, procurando que la misma se efectuara en el seno de las mismas instituciones, en espacios cedidos para tal efecto. En todos los casos, se informó a los sujetos de los objetivos del estudio, invitándolos a responder (previa firma del correspondiente consentimiento informado) un cuadernillo integrado por una hoja de datos personales y por los reactivos que se describen a continuación. El protocolo de investigación fue aprobado por la Comisión de Ética Aplicada a la Investigación Científica de la institución de pertenencia de los autores, por lo que fueron atendidos y respetados los derechos fundamentales de las personas en lo que hace a su dignidad, privacidad, confidencialidad, autonomía, autodeterminación e integridad física, mental y social.

\section{Análisis de los datos}

Antes de contrastar las hipótesis formuladas, se efectuaron algunas pruebas preliminares. En primer lugar, se examinó la presencia de potenciales sesgos debidos a la varianza del método común, mediante la prueba de un solo factor de Harman (Podsakoff, MacKenzie, Lee, \& Podsakoff, 2012), habida cuenta de que los datos fueron recolectados con instrumentos autodescriptivos. En segundo lugar, se examinó el ajuste del modelo de medida propuesto (conformado por cinco factores latentes correlacionados) con otros modelos alternativos. Para probar las hipótesis planteadas, 
se efectuó un análisis de ecuaciones estructurales, siguiendo el procedimiento recomendado por Holmbeck (2003). En un primer paso, se calcularon los parámetros correspondientes a un modelo (modelo A) integrado por las variables independientes (maquiavelismo, narcisismo y psicopatía), dependiente (satisfacción laboral), y control (sexo, edad y antigüedad laboral). En un segundo paso se incluyó, además, al hipotético mediador $(\mathrm{CO})$, restringiendo a cero los efectos directos de las variables independientes (modelo B) sobre la satisfacción laboral. Finalmente, se ajustó un tercer modelo (modelo $\mathrm{C}$ ) en el que, a diferencia del anterior, no se impusieron restricciones en ninguno de los parámetros. Seguidamente, se calculó la prueba de diferencia entre los estadísticos chi cuadrado $\left(\chi^{2}\right)$ correspondientes al modelo restringido (modelo B) y no restringido (modelo C), a fin de examinar la presencia de diferencias significativas entre ambos. Para determinar la existencia de mediación, se compararon los parámetros correspondientes a los efectos de la tríada oscura sobre la satisfacción laboral (modelos A y C). De existir mediación, la relación significativa observada en A resultaría no significativa en C (o al menos disminuiría), cuando el mediador $\mathrm{CO}$ se incluyera en la ecuación. Complementariamente, se realizaron análisis con el test de Sobel para determinar la significatividad de los efectos indirectos (Preacher, 2015). Para la estimación de los parámetros se utilizó el método de estimación de máxima verosimilitud. Para calcular el grado de ajuste del modelo mediador postulado, se calcularon los siguientes estadísticos: chi cuadrado relativo $\left(\chi^{2} / \mathrm{gl}\right.$ : Chi square degrees of freedom ratio), índice de bondad de ajuste (GFI: Goodness of Fit Index) y su variante ajustada (AGFI: Adjusted Goodness of Fit Index), índice de ajuste comparativo (CFI: Comparative Fit Index), índice de ajuste incremental (TLI: Tucker Lewis Index) y el error de aproximación de la raíz cuadrada media (RMSEA: Root Mean Square Error of Approximation).

\section{Resultados}

En la Tabla 1, se presentan medias, desviaciones estándar, pruebas de diferencia de medias ( $\mathrm{t}$ de Student) para género y correlaciones de Pearson entre las variables.

\section{Tabla 1}

Índices descriptivos, test t para género, coeficientes alpha y correlaciones de Pearson entre las variables

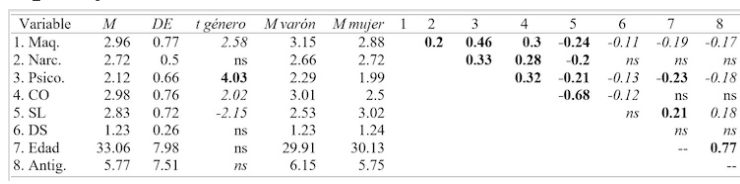

Nota: Maq (Maquiavelismo); Narc.

(Narcisismo); Psico. (Psicopatía); CO (Cinismo Organizacional); SL (Satisfacción Laboral);

DS (Deseabilidad Social); Antig. (Antigüedad

Laboral); negrita: $p<0.01$; cursiva: $p<0.05$.

La Tabla 1 muestra que las variables psicológicas correlacionaron significativamente en las direcciones previstas. Maquiavelismo, narcisismo y psicopatía correlacionaron positivamente entre sí, al tiempo que también se relacionaron positivamente con el CO. En cuanto a la satisfacción laboral, todas las variables presentaron asociaciones negativas, aunque fue en torno al cinismo donde se registraron los coeficientes más elevados.

En lo que hace al plan de análisis previsto, se llevó a cabo la prueba de Harman mediante un análisis factorial exploratorio. Todos los ítems fueron incluidos en el análisis y se forzó la extracción de un solo factor. El porcentaje de la varianza explicada por este único factor fue tan solo del $15.1 \%$, demostrando que el sesgo del método común no había afectado a los datos en estudio. Por su parte, el AFC para un modelo unifactorial mostró un ajuste débil $\left(\chi^{2} / g l=5.24, \mathrm{GFI}=0.41 ; \mathrm{AGFI}=0.4 ; \mathrm{CFI}\right.$ $=0.43 ;$ TLI $=0.42 ;$ RMSEA $=0.96)$, al igual que el AFC calculado para un modelo en el que la tríada oscura fue tratada como una escala unidimensional, o sea, sin discriminar entre maquiavelismo, narcisismo y psicopatía $\left(\chi^{2} / g l=\right.$ $5.74, \mathrm{GFI}=0.63 ; \mathrm{AGFI}=0.6 ; \mathrm{CFI}=0.59$; $\mathrm{TLI}$ $=0.57 ;$ RMSEA $=0.85)$. En cambio, el modelo penta-factorial mostró valores satisfactorios en 
los diversos índices de ajuste evaluados $\left(\chi^{2} / g l=\right.$ 2.24, GFI $=0.9, \mathrm{AGFI}=0.91, \mathrm{CFI}=0.9$, TLI $=0.9$, RMSEA $=0.03$ ).

En lo que hace a la estrategia analítica escogida para examinar los efectos directos e indirectos de la personalidad oscura sobre el $\mathrm{CO}$ y la satisfacción laboral, los parámetros calculados en el modelo A resultaron significativos solo para maquiavelismo, narcisismo y psicopatía. Estos resultados demostraron el cumplimiento de una las condiciones básicas del proceso de mediación, a saber, la preexistencia de una relación susceptible de ser mediada (Holmbeck, 2003). Concretamente, la tríada oscura explicó el $23.3 \%\left(\Delta R^{2}=0.229\right)$ de la varianza de la satisfacción laboral, proviniendo los aportes más significativos del maquiavelismo $(\#=-0.27$; $p<0.01)$, seguidos por la psicopatía $(\#=$ $-0.22 ; p<0.05)$ y el narcisismo $(\#=-0.17 ; p$ $<0.05)$, respectivamente. Las variables control (sexo, edad y antigüedad laboral) no mostraron efectos significativos sobre la satisfacción laboral.

Los parámetros calculados en el modelo B también resultaron significativos indicando tanto un impacto positivo de la tríada oscura sobre el $\mathrm{CO}$, como un efecto negativo del cinismo sobre la satisfacción laboral. En el primer caso, el mayor aporte predictivo provino del maquiavelismo (\# $=0.39 ; p<0.01)$, seguido por la psicopatía $(\#=0.22 ; p<0.01)$ y el narcisismo $(\#=$ 0.14; $p<0.05)$, respectivamente. En conjunto, los rasgos oscuros explicaron el $47.1 \%\left(\Delta R^{2}=\right.$ 0.471 ) de la varianza del CO. Tales resultados confirmaron la Hipótesis 1, mostrando que la tríada oscura es un predictor idóneo del $\mathrm{CO}$. El modelo $\mathrm{B}$ presentó una relación negativa entre el mediador y la variable dependiente, demostrado el cumplimiento de otra de las condiciones fundamentales que subyacen al proceso de mediación. Más precisamente, el CO demostró tener un impacto elevado y negativo sobre los niveles de satisfacción ( $\#=$ $-0.63 ; p<0.01)$. Una vez más, las variables control no mostraron efectos significativos sobre las variables endógenas, razón por la cual fueron omitidas en los análisis posteriores. En comparación con el modelo A, el modelo
B mostró mayor capacidad explicativa de la satisfacción laboral, aportando un $20.2 \%$ adicional a su varianza $\left(\Delta R^{2}=0.436-0.234=\right.$ 0.202).

Finalmente, el modelo $\mathrm{C}$, en el que se recalcularon los efectos directos de la tríada oscura sobre la satisfacción, no produjeron incrementos significativos en el porcentaje de varianza explicada. Un análisis pormenorizado mostró, además, que se eliminó completamente la relación directa entre la tríada oscura y la satisfacción laboral, por lo que sus efectos resultaron no significativos. Tales hallazgos, representados gráficamente en la Figura 1, indicaron una mediación total por parte del cinismo; vale decir, la totalidad de la varianza de la relación entre los rasgos oscuros y la satisfacción depende de las actitudes cínicas. Paralelamente, la prueba de diferencia entre los estadísticos $\chi^{2}$ resultó no significativa $\left(\mathrm{D} \chi^{2}=\right.$ 3.76, $p=0.289$ ). Tales resultados indicaron que no existían diferencias entre los modelos B y C. De modo que, los efectos directos añadidos en el último modelo, no aportaron ninguna mejora sustancial en el ajuste respecto del modelo intermedio en el que esta relación no fue considerada. Con lo que quedó comprobada la existencia de mediación.

\section{Figura 1}

Parámetros estimados correspondientes al modelo

C.

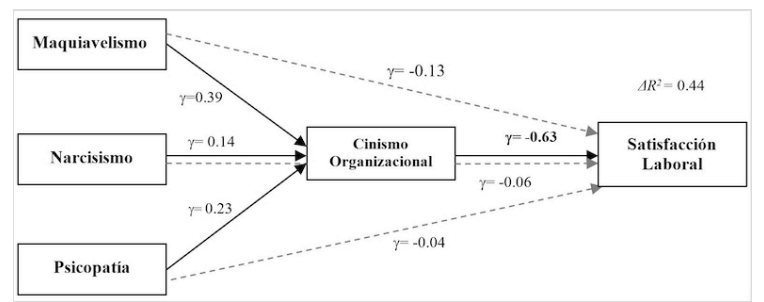

Nota. Líneas continuas: relaciones significativas; líneas cortadas: relaciones no significativas.

Para corroborar la significación de la mediación encontrada se efectuó el correspondiente test de Sobel. El mismo mostró que, en todos los casos, los efectos indirectos de la tríada oscura sobre la satisfacción eran estadísticamente significativos. Concretamente, el test mostró que la mediación del CO para el 
maquiavelismo y la psicopatía era significativa al nivel del $1 \%(z=-4.51, p<0.001 ; z=-3.24, p<$ 0.001 , respectivamente); en tanto que, en el caso del narcisismo, el nivel de probabilidad asociado al estadístico fue menor al $5 \%(z=-1.78, p<$ 0.05). Finalmente, los índices de ajuste obtenidos para el modelo mediador propuesto confirmaron su adecuación a los datos empíricos $\left(\chi^{2} / g l=1.25\right.$, $\mathrm{GFI}=0.99$; $\mathrm{AGFI}=0.98 ; \mathrm{CFI}=0.99$; $\mathrm{TLI}=$ 0.99; RMSEA $=0.02$ ). Por lo que la Hipótesis 2 quedó totalmente confirmada.

\section{Discusión}

Los resultados de la presente investigación contribuyen, en primer lugar, a clarificar el papel que desempeñan los aspectos más oscuros de la personalidad sobre el desarrollo de actitudes cínicas hacia la organización a la que se pertenece. La verificación empírica realizada proporciona apoyo total a la Hipótesis 1, demostrando que, en conjunto, los factores que conforman la tríada oscura explican una porción muy importante del cinismo organizacional (47\%). Específicamente, la suspicacia propia de los maquiavélicos (que induciría sospechas respecto a las motivaciones ocultas tras las acciones y decisiones organizacionales), la provocación psicopática (que favorecería conductas sarcásticas y maliciosas) y la grandiosidad típica de los narcisistas (que reforzaría los sentimientos de superioridad moral), conformarían un "caldo de cultivo" propiciatorio para el surgimiento y desarrollo de actitudes negativas, tales como el CO (Jones \& Figueredo, 2013; Jones \& Paulhus, 2017; Stead \& Fekken, 2014). La capacidad explicativa de consecuencias organizacionalmente no deseadas por parte de la tríada oscura coincide con resultados previamente comunicados. En este sentido, numerosos estudios (Furnham et al., 2013; Guenole, 2014; Linstead et al., 2014; O'Boyle et al., 2012; Palaiou et al., 2016; Spain et al., 2013) han subrayado que tales rasgos se encuentran asociados significativamente a déficits en el desempeño laboral, a comportamientos contraproducentes para la organización y sus miembros, a conflictos interpersonales, cinismo, cansancio emocional y falta de compromiso en el trabajo.

En segundo lugar, el presente trabajo permite una toma de posición respecto al rol que juega el CO en las asociaciones entre la tríada oscura y la satisfacción laboral. Los hallazgos obtenidos proporcionan pleno apoyo a la Hipótesis 2, demostrando que el cinismo interviene mediando totalmente tales relaciones. De acuerdo a tales resultados, el impacto de los rasgos oscuros sobre la satisfacción laboral no es lineal, sintonizando así con las conclusiones de recientes estudios (Hirschi \& Jaensch, 2015; Jonason et al., 2015; Smith \& Webster, 2017) que indican que los rasgos sombríos afectan indirectamente resultados clave como la satisfacción laboral y el desempeño a través de su acción sobre terceras variables (por ejemplo, percepciones de autoeficacia, control, autonomía, competitividad laboral, etc.).

A la luz de estos hallazgos, es posible concluir que la tríada oscura no conduce directamente a experiencias de satisfacción o insatisfacción laboral, sino a través de la promoción de actitudes negativas que afectan el modo como las personas piensan, sienten y actúan en su lugar de trabajo. En el caso concreto del $\mathrm{CO}$, se ha subrayado (Salessi \& Omar, 2014a) que el estado de suspicacia que los caracteriza conlleva un incremento de los afectos negativos. Tales estados emocionales actúan como verdaderos "tóxicos", conduciendo a experiencias de alienación laboral, despersonalización y extrañamiento, así como a un menor compromiso con la organización (Palaiou et al., 2016). Al mismo tiempo, la actitud insensible y sarcástica de los cínicos propicia una espiral de negatividad ascendente que, paulatinamente, erosiona las relaciones con los compañeros de trabajo. Como contraparte, las escasas oportunidades de desarrollo profesional (justificadas en la actitud apática y poco comprometida), sumadas a las actitudes defensivas que ellos mismos provocan en el resto de los miembros de la organización, terminan retroalimentando el circuito cínico (Brandes \& Das, 2006; Salessi \& Omar, 2014a). Así, en un escenario signado por la desconfianza, 
el pesimismo y la falta de deseo de ser parte de la organización en la que se trabaja, la experiencia de satisfacción laboral no encuentra cabida, cediendo su lugar a actitudes negativas y comportamientos contraproducentes.

\section{Implicancias prácticas}

Esta investigación tiene implicancias prácticas tanto para selectores de personal como para profesionales dedicados al desarrollo de fortalezas ejecutivas (coaching, por ejemplo). Los profesionales encargados de las áreas de evaluación y selección de personal deberían estar especialmente prevenidos para detectar elevados rasgos maquiavélicos. Sobre todo, porque durante las entrevistas de selección, estos suelen aparecer como personas atractivas para ocupar cargos de cierta importancia gerencial por su gran capacidad de persuasión, negociación, control emocional y asertividad. En cuanto a los profesionales encargados del coaching y del desarrollo de personas que podrían ocupar roles de liderazgo dentro del grupo laboral, deberían familiarizarse con la evaluación de los rasgos sombríos de la personalidad antes de iniciar su tarea. En este sentido, antes de volcar sus esfuerzos en el desarrollo de habilidades de conducción, incluyendo actitudes positivas, adaptabilidad y efectividad, deberían identificar el lado oscuro de la personalidad para tratar de mitigar el impacto de tales características sobre los comportamientos y actitudes negativas (tales como CO) del líder dentro del grupo.

\section{Limitaciones y sugerencias para futuras investigaciones}

Como toda investigación empírica, el presente estudio presenta algunas limitaciones que merecen ser comentadas. Al respecto, hay que subrayar que, puesto que el procedimiento de selección muestral no fue aleatorio, la generalización de los resultados a la población de trabajadores argentinos debería hacerse con prudencia y cautela. Sin embargo, con el propósito de minimizar este inconveniente, se procuró que la muestra en estudio incluyera empresas e instituciones de los más diferentes rubros y niveles de complejidad organizacional. Otra limitación podría estar vinculada con el diseño transversal utilizado, lo que limita cualquier inferencia de causalidad entre la personalidad oscura y el resto de las variables estudiadas. En futuras investigaciones, el empleo de diseños longitudinales sería particularmente útil para establecer el orden causal de las relaciones encontradas en el presente trabajo. A pesar de tales limitaciones, los resultados obtenidos contribuyen al conocimiento del papel que desempeña el $\mathrm{CO}$ en las relaciones entre los aspectos sombríos de la personalidad y los sentimientos de insatisfacción en el trabajo.

Por las innegables consecuencias sobre la salud y el bienestar de las organizaciones y de sus miembros, cualquier agenda para futuros estudios debería contemplar la posibilidad de seguir profundizando sobre el impacto laboral de los rasgos oscuros de la personalidad. En este sentido, sería importante examinar los efectos de otros rasgos disfuncionales de personalidad, como los contenidos en las taxonomías propuestas por Guenole (2014) y Hogan y Hogan (2009). Asimismo, como la globalización sigue expandiéndose, y las compañías siguen trasplantando gerentes desde sus casas matrices hacia los más recónditos lugares del mundo, sería conveniente explorar las posibles vinculaciones entre los rasgos oscuros de la personalidad y las prácticas de gerenciamiento que implementan tales líderes organizacionales (Omar \& Urteaga, 2010). Asimismo, sería muy interesante analizar si la cultura nacional (manifestada a través de los valores personales) tiene la potencialidad de crear "nichos ocupacionales" (gerencias, áreas de supervisión estratégica y similares) capaces de darle cobijo a las personalidades más sombrías. El mayor conocimiento de la sinergia entre los aspectos culturales y la personalidad oscura no solo redundaría en una aprehensión más holística de la situación, sino que también favorecía la implementación de programas de mejora e intervenciones más eficaces para todos. 


\section{Referencias}

Aly, N., Ghanem, M., \& El-Shanawany, S. (2016). Organizational cynicism and its consequences on nurses and quality of care in critical care and toxicology units. Journal of Education and Practice, 7(8), 85-96. (N. ${ }^{\circ}$ de servicio de reproducción de documentos ERIC EJ 1095331)

Brandes, P., \& Das, D. (2006). Locating behavioral cynicism at work: Construct issues and performance implications. En P. L. Perrewé \& D. C. Ganster (Eds.), Employee health, coping and methodologies (pp. 233-266). Philidelphia, PA: Elsevier Science.

Chiaburu, D. S., Peng, A. C., Oh, I., Banks, G. C., $\&$ Lomeli, L. C. (2013). Antecedents and consequences of employee organizational cynicism: A meta-analysis. Journal of Vocational Behavior, 83(2), 181-197. https:/ /doi.org/10.1016/j.jvb.2013.03.007

Furnham, A., Richards, S. C., \& Paulhus, D. L. (2013). The dark triad of personality: A 10 year review. Social and Personality Psychology Compass, 7(3), 199-216. https://doi.org/10. $1111 / \mathrm{spc} 3.12018$

Guenole, N. (2014). Maladaptive personality at work: Exploring the darkness. Industrial $\mathbb{E}$ Organizational Psychology, 7(1), 85-97. http s://doi.org/10.1111/iops.12114

Hirschi, A., \& Jaensch, V. (2015). Narcissism and career success: Occupational self-efficacy and career engagement as mediators. Personality and Individual Differences, 77, 205-208. https://doi.org/10.1016/j.paid.201 5.01 .002

Hogan, R., \& Hogan, J. (2009). Hogan development survey manual (2.a ed.). Tulsa, OK: Hogan Assessment Systems.

Holmbeck, G. (2003). Toward terminological, conceptual, and statistical clarity in the study of mediators and moderators. En A. E. Kazdin (Ed.), Methodological issues and strategies in clinical research (3.a ed., pp. 77-105). Washington, DC: American Psychological Association.
Jones, D. N., \& Figueredo, A. J. (2013). The core of darkness: Uncovering the heart of the Dark Triad. European Journal of Personality, 27(6), 521-531. https://doi.org/10.1002/per .1893

Jones, D. N., \& Paulhus, D. L. (2014). Introducing the Short Dark Triad (SD3): A brief measure of dark personality traits. Assessment, 21(1), 28-41. https://doi.org/10 $.1177 / 1073191113514105$

Jones, D. N., \& Paulhus, D. L. (2017). Duplicity among the dark triad: Three faces of deceit. Journal of Personality and Social Psychology, 113, 329-342. https://doi.org/10.1037/pspp 0000139

Jonason, P., Weeb, S., \& Li, N. (2015). Competition, autonomy, and prestige: Mechanisms through which the Dark Triad predict job satisfaction. Personality and Individual Differences, 72, 112-116. https://d oi.org/10.1016/j.paid.2014.08.026

Judge, T., Weiss, H., Kammeyer-Mueller, J., \& Hulin, C. (2017). Job attitudes, job satisfaction, and job affect: A century of continuity and of change. The Journal of Applied Psychology, 102, 356-374. https://do i.org/10.1037/apl0000181

Linstead, S., Maréchal, M., \& Griffin, R. W. (2014). Theorizing and researching the dark side of organization. Organization Studies, 35(2), 165-188. https://doi.org/10.1 177/0170840613515402

Michel, J., \& Bowling, N. (2013). Does dispositional aggression feed the narcissistic response? The role of narcissism and aggression in the prediction of job attitudes and counterproductive work behaviors. Journal of Business and Psychology, 28(1), 93-105. https://doi.org/10.1007/s10869-01 2-9265-6

O’Boyle, H., Forsyth, D., Banks, G., \& McDaniel, M. (2012). A meta-analysis of the Dark Triad and work behavior: A social exchange perspective. Journal of Applied Psychology, 97(3), 557-579. https://doi.org/10.1037/a0 025679

Omar, A. (1988). Estandarización argentina de los cuestionarios de personalidad 
de Eysenck. Revista Chilena de NeuroPsiquiatría, 26(2), 83-95. Recuperado de https://www.researchgate.net/publicatio n/274383019_Estandarizacion_Argentina _de_los_Cuestionrios_de_Personalidad_de Eysenck

Omar, A., \& Urteaga, F. (2010). El impacto de la cultura nacional sobre la cultura organizacional. Universitas Psychologica, 9(1), 79-92. Recuperado de http://revistas.javeriana.edu.co/index.p hp/revPsycho/article/view/276

Palaiou, K., Zarola, A., \& Furnham, A. (2016). The dark side of personality predicts positive and negative work attitudes. Personality $\mathcal{E}$ Individual Differences, 88, 12-16. https://doi.org/10.1016/j.paid.2015. 08.029

Palmer, J., Komarraju, M., Carter, M., \& Karau, S. (2017). Angel on one shoulder: Can perceived organizational support moderate the relationship between the Dark Triad traits and counterproductive work behavior? Personality $\mathbb{E}$ Individual Differences, 110, 31-37. https://doi.org/10.1 016/j.paid.2017.01.018

Paulhus, D. L. (2014). Toward a taxonomy of dark personalities. Current Directions in Psychological Science, 23(6), 421 - 426. https ://doi.org/0.1177/0963721414547737

Podsakoff, P. M., MacKenzie, S. B., Lee, J., \& Podsakoff, N. P. (2012). Sources of method bias in social science research and recommendations on how to control it. Annual Review of Psychology, 63(1), 539-569. https://doi.org/10.1146/annurevpsych-120710-100452

Preacher, K. J. (2015). Advances in mediation analysis: A Survey and synthesis of new developments. Annual Review of Psychology, 66(6), 825-852. https://doi.org/10.1146/an nurev-psych-010814-015258

Salessi, S., \& Omar, A. (2014a). Cinismo organizacional: consecuencias sobre la salud mental de los trabajadores. Psicología y Salud, 24(2), 269-277. Recuperado de http://revistas.uv.mx/index.php/psicysal ud/article/view/930
Salessi, S., \& Omar, A. (2014b). Validación de la Escala de Cinismo Organizacional: un estudio con trabajadores argentinos. Revista de Psicología, 32(2), 357-385. Recuperado de http://revistas.pucp.edu.pe/index.php/ps icologia/article/view/10953/11463

Salessi, S., \& Omar, A. (2016). Satisfacción laboral genérica: propiedades psicométricas de una escala para medirla. Revista Alternativas en Psicología, 34, 93-108. Recuperado de https://alternativas.me/attachments/arti cle/116/8\%20-\%20Satisfacci\%C3\%B3n\%2 OLaboral\%20Gen\%C3\%A9rica.pdf

Salessi, S., \& Omar, A. (en prensa). Psychometric properties of a scale to measure the dark side of personality. Estudos de Psicología,

Smith, M., \& Webster, B. (2017). A moderated mediation model of Machiavellianism, social undermining, political skill, and supervisor-rated job performance. Personality and Individual Differences, 104, 453-459. https://doi.org/10.1016/j.paid.201 6.09.010

Spain, S. M., Harms, P., \& Lebreton, J. M. (2013). The dark side of personality at work. Journal of Organizational Behavior, 35, 41-60. https: //doi.org/10.1002/job.1894

Spurk, D., Keller, A., \& Hirschi, A. (2016). Do bad guys get ahead or fall behind? Relationships of the Dark Triad of personality with objective and subjective career success. Social Psychological and Personality Science, 7, 113-121. https://doi.o $\mathrm{rg} / 10.1177 / 1948550615609735$

Stead, R., \& Fekken, G. (2014). Agreeableness at the core of the dark triad of personality. Individual Differences Research, 12(4A), 131-141. Recuperado de http://en.buaa.findplus.cn/?h=articles $\&$ $\mathrm{db}=\mathrm{a} 9 \mathrm{~h} \& \mathrm{an}=100210820 \#$

Sundstrom, E., Lounsbury, J., Gibson, L., \& Huang, J. (2016). Personality traits and career satisfaction in training and development occupations: Toward a distinctive T\&D personality profile. Human Resource Development Quarterly, 27, 13-40. https://doi.org/10.1002/hrdq.21223 
Thundiyil, T., Chiaburu, D., In-Sue, O., Banks, G., \& Peng, A. (2014). Cynical about change? A metaanalysis of organizational cynicism correlates. Academy of Management Meeting Proceedings, 783-786. https://doi.org/10.546 5/AMBPP.2014.177

\section{Notas}

* Artículo de investigación. Esta investigación fue financiada por el Consejo Nacional de Investigaciones Científicas y Técnicas (CONICET), Argentina, en el marco de la beca postdoctoral otorgada a la primera autora, bajo la dirección de la segunda. 